\title{
Observational constraints on braneworld inflation: the effect of a Gauss-Bonnet term
}

\author{
Shinji Tsujikawa ${ }^{1}$, M. Sami ${ }^{2}$ and Roy Maartens ${ }^{3}$ \\ 1 Department of Physics, Gunma National College of Technology, Gunma 371-8530, Japan \\ 2 IUCAA, Post Bag 4, Ganeshkhind, Pune 411 007, India and \\ 3 Institute of Cosmology \& Gravitation, University of Portsmouth, Portsmouth PO1 2EG, UK
}

(Dated: February 2, 2008)

\begin{abstract}
High-energy modifications to general relativity introduce changes to the perturbations generated during inflation, and the latest high-precision cosmological data can be used to place constraints on such modified inflation models. Recently it was shown that Randall-Sundrum type braneworld inflation leads to tighter constraints on quadratic and quartic potentials than in general relativity. We investigate how this changes with a Gauss-Bonnet correction term, which can be motivated by string theory. Randall-Sundrum models preserve the standard consistency relation between the tensor spectral index and the tensor-to-scalar ratio. The Gauss-Bonnet term breaks this relation, and also modifies the dynamics and perturbation amplitudes at high energies. We find that the GaussBonnet term tends to soften the Randall-Sundrum constraints. The observational compatibility of the quadratic potential is strongly improved. For a broad range of energy scales, the quartic potential is rescued from marginal rejection. Steep inflation driven by an exponential potential is excluded in the Randall-Sundrum case, but the Gauss-Bonnet term leads to marginal compatibility for sufficient e-folds.
\end{abstract}

\section{INTRODUCTION}

The WMAP, SDSS and other data of high-precision cosmology open up the possibility of testing and constraining models of the universe (see e.g. Ref. [1]). The standard model based on general relativity and inflation passes all current observational tests with suitable proportions of dark energy and dark matter, and without the need for departures from adiabaticity, gaussianity or scale-invariance in the primordial perturbations. This gives strong support to the slow-roll inflationary scenario, while at the same time initiating a period in which the inflationary parameters can be constrained by observations. Indeed, the viable slow-roll parameter space is dramatically reduced 2,3$]$.

It is not possible to reconstruct the functional form of the inflationary potential from observations. However, improved constraints may be derived if a general form of the potential is assumed, and if appropriate limits are placed on the number $N$ of e-folds of inflation after horizon-crossing. Some inflationary potentials are under pressure from the data, with the chaotic quartic potential $\left(V \propto \phi^{4}\right)$ marginally ruled out at the $2 \sigma$ level [3]. Constraints on slow-roll inflationary potentials follow from the properties of their primordial perturbation spectra, and in particular from the degeneracy between scalar and tensor perturbations. This degeneracy is expressed via the standard consistency relation $R=-8 n_{\mathrm{T}}$ (discussed below). The degeneracy arises since a tensor contribution increases power on large scales, requiring a compensating increase of small-scale scalar power, and thus a more blue spectrum $n_{\mathrm{S}}>1$.

These recent advances in observational constraints on slow-roll potentials may be extended to the case where Einstein's general relativity is modified at inflationary energy scales. If the true fundamental scale of gravity is lower than the effective four-dimensional Planck scale $M_{4} \sim 10^{16} \mathrm{TeV}$, as in higher-dimensional gravity theories, then significant modifications to general relativity may arise during inflation. The simplest phenomenological models describing such a scenario are the fivedimensional Randall-Sundrum type braneworld cosmologies (see e.g. Ref. [4] for recent reviews). These models are motivated by ideas from string theory and M theory. The observable universe is a four-dimensional "brane" surface embedded in a five-dimensional anti de Sitter "bulk" spacetime, with standard model fields trapped on the brane, while gravity propagates in the bulk. Although the fifth dimension is infinite, the curvature of the bulk acts to confine the graviton near the brane at low energies, so that general relativity is recovered. At high energies in the early universe, graviton localization fails and the Friedmann equation is modified. The amplitudes and spectra of perturbations generated during inflation are also modified [5, 6 .

Remarkably, despite the modifications to both scalar and tensor primordial perturbations, the consistency relation $R=-8 n_{\mathrm{T}}$ is unchanged in this braneworld scenario 7]. (We may expect some change from higher-order expansions in slow-roll parameters 8], but this modification is too small to be detected in current observations, and it is also not clear that bulk perturbations may be neglected at higher order.) As a consequence, observational constraints on the primordial perturbation spectra cannot distinguish between the general relativistic and braneworld models 9]. Braneworld effects on the evolution of perturbations after horizon re-entry will leave signatures on the cosmic microwave background (CMB), but the techniques for calculating these signatures are still under development [10]. If constraints are only avail- 
able from the primordial perturbation spectra, then specific braneworld parameters cannot be constrained - but the constraints on particular slow-roll potentials are modified. For example, the perturbations produced by the quadratic potential $\left(V \propto \phi^{2}\right)$ are further from scaleinvariance than in the standard cosmology, while in the quartic case they are more or less unchanged [11, 12]. Thus both of the simplest chaotic potentials are under strong observational pressure in the simplest braneworld scenario.

The Randall-Sundrum braneworld cosmology is based on the five-dimensional Einstein-Hilbert action. At high energies, it is expected that this action will acquire quantum corrections. String theory and holography indicate that higher-order curvature invariants will arise in the action at perturbative level. In five dimensions, the Gaussbonnet curvature invariant has special properties: it is the unique combination that leads to second-order field equations linear in the second derivatives, and it is ghostfree (see e.g. Refs. [13, 14, 15]). A natural question is whether the Gauss-Bonnet correction to the EinsteinHilbert action will disrupt the degeneracy between tensor and scalar perturbations. The answer is that the standard consistency relation is broken by the Gauss-Bonnet term [16].

However, the breaking of degeneracy is "mild", and it turns out, as we shall show, that the likelihood values are almost identical to those in the standard and Randall-Sundrum cases. Thus the introduction of a Gauss-Bonnet term does not lead to braneworld signatures in current observations of the primordial perturbation spectra. However, it does lead to interesting changes in the constraints on inflationary potentials. The quadratic potential moves inside the $1 \sigma$ bound for a range of inflationary energy scales even for $N=50$, so that the simplest chaotic inflation model is "rescued" by the Gauss-Bonnet correction. The quartic potential is also removed from marginal rejection, and is inside the $2 \sigma$ bound for a range of energy scales even when $N=50$. The exponential potential, which can drive "steep" inflation in Randall-Sundrum models [17], is excluded by observations in the Randall-Sundrum case. The GaussBonnet correction again rescues this potential, moving it inside the $2 \sigma$ bound for a range of energy scales when $N \gtrsim 55$.

\section{INFLATION IN A GAUSS-BONNET BRANEWORLD}

For a 5D bulk with Einstein-Gauss-Bonnet gravity, containing a $4 \mathrm{D}$ brane, the gravitational action is

$$
\begin{aligned}
\mathcal{S}= & \frac{1}{2 \kappa_{5}^{2}} \int d^{5} x \sqrt{-{ }^{(5)} g}\left[-2 \Lambda_{5}+\mathcal{R}\right. \\
& \left.+\alpha\left(\mathcal{R}^{2}-4 \mathcal{R}_{a b} \mathcal{R}^{a b}+\mathcal{R}_{a b c d} \mathcal{R}^{a b c d}\right)\right] \\
& -\int_{\text {brane }} d^{4} x \sqrt{-g} \sigma
\end{aligned}
$$

where $x^{a}=\left(x^{\mu}, y\right)$, with $y$ the extra-dimensional coordinate, $g_{a b}={ }^{(5)} g_{a b}-n_{a} n_{b}$ is the induced metric, with $n^{a}$ the unit normal to the brane, $\sigma(>0)$ is the brane tension, and $\Lambda_{5}(<0)$ is the bulk cosmological constant. The fundamental energy scale of gravity is the $5 \mathrm{D}$ scale $M_{5}$, where $\kappa_{5}^{2}=8 \pi / M_{5}^{3}$. The Planck scale $M_{4}$ is an effective scale, describing gravity on the brane at low energies, and typically $M_{4} \gg M_{5}$.

The Gauss-Bonnet (GB) term may be thought of as the lowest-order stringy correction to the 5D EinsteinHilbert action, with coupling constant $\alpha \geq 0$. In this case, $\alpha\left|\mathcal{R}^{2}\right| \ll|\mathcal{R}|$, so that $\alpha \ll \ell^{2}$, where $\ell$ is the bulk curvature scale, $|\mathcal{R}| \sim \ell^{-2}$. In terms of the extradimensional energy scale $\mu \equiv \ell^{-1}$,

$$
\beta \equiv 4 \alpha \mu^{2} \ll 1
$$

For an anti de Sitter bulk, $\Lambda_{5}=-3 \mu^{2}(2-\beta)$. The Randall-Sundrum (RS) type models are recovered for $\beta=0$.

Imposing $Z_{2}$ symmetry across a Friedmann brane in an anti de Sitter bulk, we get the Friedmann equation [13, 15

$$
\begin{aligned}
H^{2} & =\frac{\mu^{2}}{\beta}\left[(1-\beta) \cosh \left(\frac{2 \chi}{3}\right)-1\right], \\
\kappa_{5}^{2}(\rho+\sigma) & =2 \mu\left[\frac{2(1-\beta)^{3}}{\beta}\right]^{1 / 2} \sinh \chi,
\end{aligned}
$$

where $H$ is the Hubble rate, and $\chi$ is a dimensionless measure of the energy density $\rho$ on the brane. Eliminating $\chi$ leads to

$$
\kappa_{5}^{2}(\rho+\sigma)=2 \mu \sqrt{1+\frac{H^{2}}{\mu^{2}}}\left[3-\beta+2 \beta \frac{H^{2}}{\mu^{2}}\right] .
$$

In order to recover General Relativity (GR) at low energies, the effective 4D Newton constant is [13, 14]

$$
\kappa_{4}^{2}=\frac{\mu}{1+\beta} \kappa_{5}^{2}
$$

The brane tension is fine-tuned by achieving a zero cosmological constant on the brane [14]:

$$
\kappa_{5}^{2} \sigma=2 \mu(3-\beta)
$$

By Eqs. (3) and (4) there is a characteristic GB energy scale,

$$
m_{\beta}=\left[\frac{8 \mu^{2}(1-\beta)^{3}}{\beta \kappa_{5}^{4}}\right]^{1 / 8},
$$

such that the GB high energy regime $(\sinh \chi \gg 1)$ corresponds to $\rho \gg m_{\beta}^{4}$. Since the GB term is a correction to the RS action, we must have $m_{\beta}^{4}$ greater than the RS energy scale $\sigma$; this requires [16] $\beta<0.152$, which is consistent with Eq. (2). Expanding Eq. (3) in $\chi$, we 
find three regimes for the dynamical history of the brane universe [16]

$$
\begin{aligned}
& \rho \gg m_{\beta}^{4} \Rightarrow H^{2} \approx\left[\frac{\mu^{2} \kappa_{5}^{2}}{4 \beta} \rho\right]^{2 / 3} \quad \text { (GB regime) },(9) \\
& m_{\beta}^{4} \gg \rho \gg \sigma \Rightarrow H^{2} \approx \frac{\kappa_{4}^{2}}{6 \sigma} \rho^{2} \quad(\text { RS regime }), \quad(10) \\
& \rho \ll \sigma \Rightarrow H^{2} \approx \frac{\kappa_{4}^{2}}{3} \rho \quad(\text { GR regime). }
\end{aligned}
$$

We are mainly interested in inflation which occurs in the GB regime, Eq. (9), and in the intermediate regime between Eqs. (9) and (10). These are the regimes where the GB correction is crucial. We assume a 4D inflaton field $\phi$ with potential $V(\phi)$ on the brane, which satisfies the Klein-Gordon equation,

$$
\ddot{\phi}+3 H \dot{\phi}+V_{\phi}(\phi)=0 .
$$

During slow-roll inflation, $|\ddot{\phi}| \ll|3 H \dot{\phi}|$ and $\dot{\phi}^{2} \ll V(\phi)$. Since $V \approx \rho \gg \sigma$, Eq. (4) gives $V=m_{\beta}^{4} \sinh \chi$. Making use of Eq. (3), we find that the slow-roll parameters, $\epsilon \equiv$ $-\dot{H} / H^{2}$ and $\eta \equiv V_{\phi \phi} / 3 H^{2}$, are given by

$$
\begin{aligned}
\frac{\epsilon}{\epsilon_{\mathrm{RS}}} & =\frac{2(1-\beta)^{4} \sinh \frac{2}{3} \chi \tanh \chi \sinh ^{2} \chi}{9(1+\beta)(3-\beta)\left[(1-\beta) \cosh \frac{2}{3} \chi-1\right]^{2}}, \\
\frac{\eta}{\eta_{\mathrm{RS}}} & =\frac{2(1-\beta)^{3} \sinh ^{2} \chi}{3(1+\beta)(3-\beta)\left[(1-\beta) \cosh \frac{2}{3} \chi-1\right]},
\end{aligned}
$$

where $\epsilon_{\mathrm{RS}}$ and $\eta_{\mathrm{RS}}$ are the RS slow-roll parameters [5],

$$
\epsilon_{\mathrm{RS}}=\frac{2 \sigma}{\kappa_{4}^{2}} \frac{V_{\phi}^{2}}{V^{3}}, \quad \eta_{\mathrm{RS}}=\frac{2 \sigma}{\kappa_{4}^{2}} \frac{V_{\phi \phi}}{V^{2}} .
$$

In the limit $\chi \ll 1$ we have $\epsilon \rightarrow \epsilon_{\mathrm{RS}}$ and $\eta \rightarrow \eta_{\mathrm{RS}}$. The number of e-folds, $N \equiv \int H d t$, is

$$
N \approx 3 \int_{\chi_{e}}^{\chi_{N}} \frac{H^{2}}{V_{\chi}}\left(\frac{\mathrm{d} \phi}{\mathrm{d} \chi}\right)^{2} \mathrm{~d} \chi,
$$

where $\chi_{e}$ is evaluated at the end of inflation $(\epsilon=1)$, and $\chi_{N}$ is evaluated when cosmological scales leave the horizon. This value of $\chi$ is subject to a quantum gravity upper limit: in order to consistently apply semi-classical analysis of inflationary perturbations, we require $V<$ $M_{5}^{4}$, which yields the constraint 16]

$$
\sinh \chi<\left(\frac{M_{5}}{m_{\beta}}\right)^{4} .
$$

We consider simple monomial potentials corresponding to chaotic large-field inflation:

$$
V(\phi)=V_{0} \phi^{p} .
$$

We are mainly interested in the cases $p=2,4$ and $p \rightarrow \infty$ (exponential potential). The slow-roll parameters be- come

$$
\begin{aligned}
\epsilon & =\left[\frac{4 \sigma p^{2} V_{0}^{2 / p}}{27 \kappa_{4}^{2} m_{\beta}^{4+8 / p}}\right] f(\chi), \\
\eta & =\left[\frac{4 \sigma p(p-1) V_{0}^{2 / p}}{9 \kappa_{4}^{2} m_{\beta}^{4+8 / p}}\right] g(\chi),
\end{aligned}
$$

where

$$
\begin{aligned}
& f(\chi)=\frac{\sinh \frac{2}{3} \chi \tanh \chi(\sinh \chi)^{1-2 / p}}{\left[\cosh \frac{2}{3} \chi-1\right]^{2}}, \\
& g(\chi)=\frac{(\sinh \chi)^{1-2 / p}}{\cosh \frac{2}{3} \chi-1}
\end{aligned}
$$

and we used Eq. (2). In the high-energy regime $\sinh \chi \gg$ 1 , we have $f(\chi), g(\chi) \propto \exp [(p-6) \chi / 3 p]$. Therefore for $p<6$ both slow-roll parameters grow with the decrease of $\chi$. On the other hand, in the case $p>6$, including steep inflation, the situation is opposite, as pointed out in Ref. [15]. In the regime $\chi \ll 1$ one has $\epsilon, \eta \propto \chi^{-1-2 / p}$, so that both slow-roll parameters increase with decreasing $\chi$ for all positive values of $p$.

In Fig. 1 we show $f(\chi)$ for $p=2,4, \infty$. The behaviour of $g(\chi)$ is qualitatively similar. When $p \rightarrow \infty$, the slowroll parameter $\epsilon$ gets smaller with the decrease of $\chi$ in the GB regime $(\chi \gtrsim 3)$, after which it begins to grow in the RS regime. Therefore inflation comes to an end in the RS regime as argued in Ref. [15]. On the other hand $\epsilon$ always increases with the decrease of $\chi$ for $p=2$ and $p=4$, as seen in Fig. 1] This means that inflation can end either in the RS or GB regime, depending on the amplitude of the coefficient terms in Eqs. (19) and (20). In order to estimate the maximum values of $|\epsilon|$ and $|\eta|$ for cosmologically relevant scales, it is sufficient to consider the case where inflation terminates in the $\mathrm{RS}$ regime $\left(\chi_{e} \ll 1\right)$. This is because one obtains smaller values of $|\epsilon|$ and $|\eta|$ when inflation ends in the GB regime.

The number of e-folds, Eq. (16), is

$$
\begin{aligned}
N & =\frac{3 \mu^{2}}{\beta p^{2} V_{0}^{2 / p} m_{\beta}^{4-8 / p}} \int_{\chi_{e}}^{\chi_{N}} \frac{\left[\cosh \frac{2}{3} \chi-1\right] \cosh \chi}{(\sinh \chi)^{2-2 / p}} d \chi \\
& \equiv \frac{3 \mu^{2}}{\beta p^{2} V_{0}^{2 / p} m_{\beta}^{4-8 / p}}[I(\chi)]_{\chi_{e}}^{\chi_{N}} .
\end{aligned}
$$

One can find an explicit form of the integral $I(\chi)$ for particular values of $p$, as we see later.

Let us consider the case where inflation ends in the RS regime $\left(\chi_{e} \ll 1\right)$. By Eq. (23), $I(\chi) \sim 2 p \chi^{(2+p) / p} / 9(2+p)$ for $\chi \ll 1$ (setting the integration constant to zero), so that

$$
N=\frac{3 \mu^{2}}{\beta p^{2} V_{0}^{2 / p} m_{\beta}^{4-8 / p}}\left[I\left(\chi_{N}\right)-\frac{2 p}{9(2+p)} \chi_{e}^{1+2 / p}\right] .
$$

By Eq. (13) we obtain for $\chi \ll 1$

$$
\epsilon=\left[\frac{2 \sigma p^{2} V_{0}^{2 / p}}{\kappa_{4}^{2} m_{\beta}^{4+8 / p}}\right] \chi^{-1-2 / p},
$$




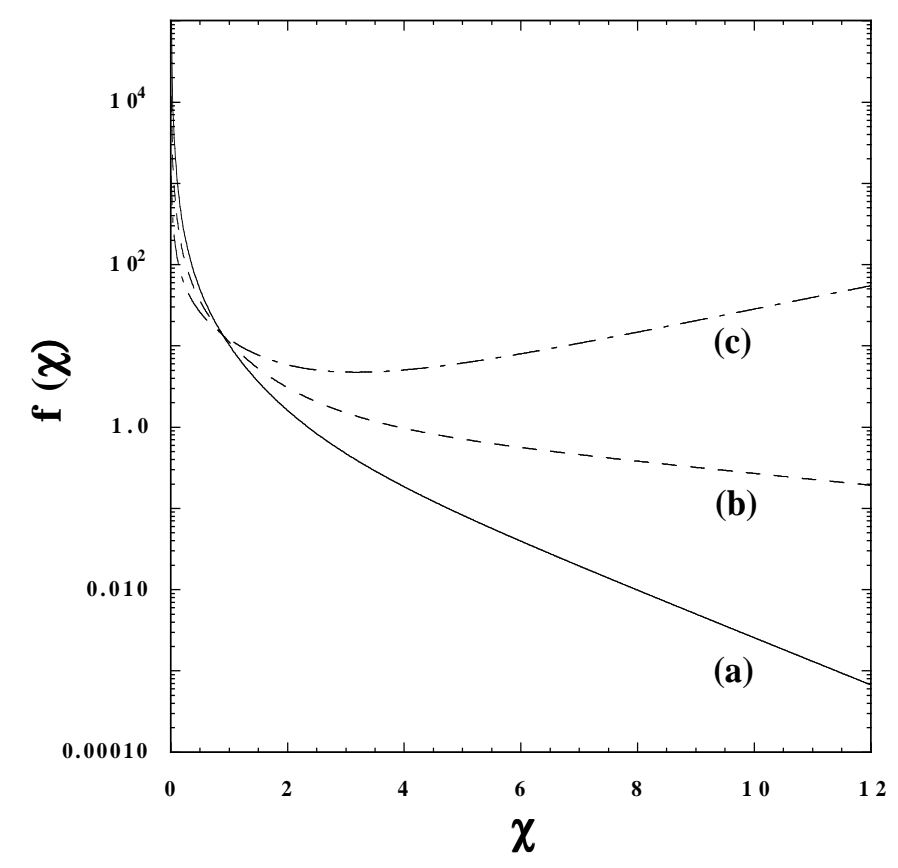

FIG. 1: The dependence of the slow-roll parameter $\epsilon$ on $\chi$ [see Eq. (19)] for (a) $p=2$, (b) $p=4$ and (c) $p \rightarrow \infty$.

so that the value of $\chi$ at the end of inflation is

$$
\chi_{e}=\frac{V_{0}}{m_{\beta}}\left(\frac{2 \sigma p^{2}}{\kappa_{4}^{2} V_{0}}\right)^{p /(p+2)} .
$$

Combining these results gives the following relation

$$
\frac{2 \sigma p^{2} V_{0}^{2 / p}}{\kappa_{4}^{2} m_{\beta}^{4+8 / p}}=\frac{9(2+p)}{2[N(2+p)+p]} I\left(\chi_{N}\right) .
$$

Then the slow-roll parameters, Eqs. (19) and (20), may be written for $\chi_{e} \ll 1$ in the form

$$
\begin{aligned}
\epsilon & =\frac{(2+p) I\left(\chi_{N}\right)}{3[N(2+p)+p]} f\left(\chi_{N}\right), \\
\eta & =\frac{(p-1)(p+2) I\left(\chi_{N}\right)}{p[N(2+p)+p]} g\left(\chi_{N}\right) .
\end{aligned}
$$

These expressions agree with Ref. [15] in the special case $p \rightarrow \infty$.

When $p=2$ the integral in Eq. (23) can be evaluated:

$$
I(\chi)=\frac{3}{2}\left[\cosh \frac{2}{3} \chi-1-\ln \left(\frac{1}{3}+\frac{2}{3} \cosh \frac{2}{3} \chi\right)\right],
$$

where the integration constant has been chosen so that $I(\chi) \sim \chi^{2} / 9$ for $\chi \ll 1$. When $p=4$ we do not have an analytical form, but the integral can be found numerically. In the limit $p \rightarrow \infty$ the integral is given by [15]

$$
I(\chi)=\frac{2}{\sqrt{3}} \tan ^{-1}\left(\frac{2}{\sqrt{3}} \sinh \frac{\chi}{3}\right)+\frac{1-\cosh \frac{2}{3} \chi}{\sinh \chi}
$$

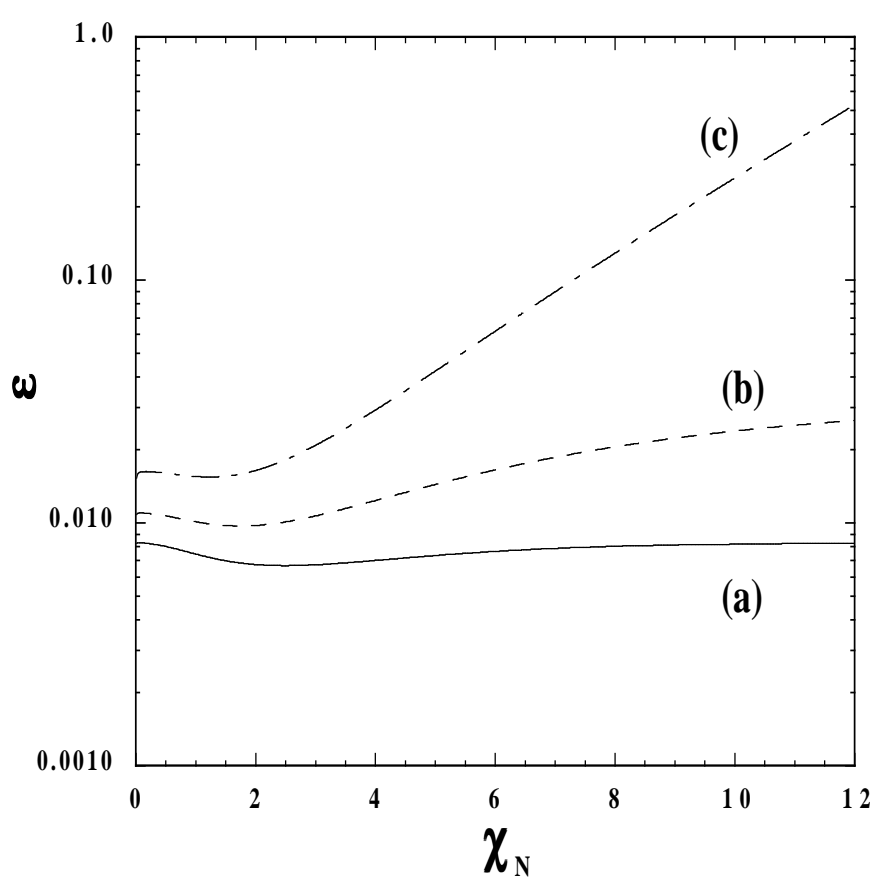

FIG. 2: The dependence of $\epsilon$ on $\chi_{N}$, for $N=60$ and (a) $p=2$, (b) $p=4$, (c) $p \rightarrow \infty$.

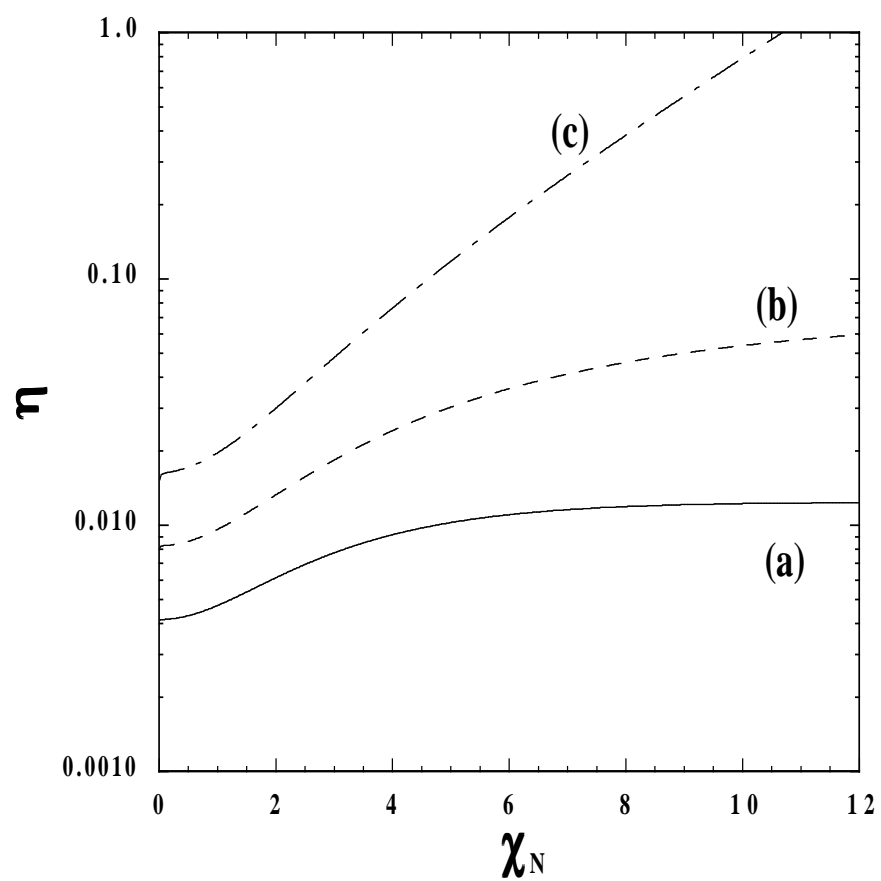

FIG. 3: The dependence of $\eta$ on $\chi_{N}$, for $N=60$ and (a) $p=$ 2 , (b) $p=4$, (c) $p \rightarrow \infty$. 
which behaves as $I(\chi) \sim 2 \chi / 9$ for $\chi \ll 1$.

In Figs. 2] and [3] we plot the slow-roll parameters as functions of $\chi_{N}$ for $p=2,4, \infty$. We integrated Eq. (23) numerically and checked that this shows excellent agreement with the analytical results for $p=2$ and $p \rightarrow \infty$. The GB $\left(\sinh \chi_{N} \gg 1\right)$ and $\mathrm{RS}\left(\chi_{N} \ll 1\right)$ regimes are connected by an intermediate regime of a combination of GB and RS inflation. Figure 2 shows that $\epsilon$ has a minimum in this region $\left(1.5 \lesssim \chi_{N} \lesssim 3\right)$. This is an important feature for estimating the ratio of tensor to scalar perturbations, as we see in the next section.

The parameter $\eta$ is a monotonically increasing function. It is also evident from Figs. 2 and 3 that both slowroll parameters increase with an increase in $p$. Note, however, that we can have $\epsilon, \eta<1$ even in the limit $p \rightarrow \infty$, which means that inflation can be realized for a steep exponential potential, as in the RS case. If we choose smaller values of $N$ than shown in Figs. 2 and 3. the slow-roll parameters tend to increase for a given value of $\chi_{N}$. The qualitative behaviour of $\epsilon$ and $\eta$ is similar to that illustrated in Figs. 2] and 3 for cosmologically relevant scales $(50 \lesssim N \lesssim 70)$.

\section{PERTURBATION SPECTRA}

In this section we evaluate the spectra of perturbations produced during slow-roll inflation and place constraints on the GB braneworld inflationary models using a compilation of the latest observational data.

The amplitude of scalar perturbations is given by 16$]$

$$
A_{\mathrm{S}}^{2}=\frac{9 H^{6}}{2 \pi^{2} V_{\phi}^{2}}=\left[\frac{\kappa_{4}^{6} V^{3}}{6 \pi^{2} V_{\phi}^{2}}\right] G_{\beta}^{2}(H / \mu)
$$

where the term in square brackets is the standard amplitude, and the GB braneworld modification is given by

$$
G_{\beta}^{2}(x)=\left[\frac{3(1+\beta) x^{2}}{2 \sqrt{1+x^{2}}\left(3-\beta+2 \beta x^{2}\right)+2(\beta-3)}\right]^{3},
$$

where $x \equiv H / \mu$ is a dimensionless measure of energy scale. The RS amplification factor [5] is recovered when $\beta=0$. For the monomial potential, we find that

$A_{\mathrm{S}}^{2}=\frac{3^{3-1 / p} \kappa_{4}^{2-2 / p} \sigma^{-1+1 / p} \mu^{4+2 / p}}{2^{3-2 / p} \pi^{2} p^{2} V_{0}^{2 / p} \beta^{2+1 / p}} \frac{\left[\cosh \frac{2}{3} \chi-1\right]^{3}}{(\sinh \chi)^{2-2 / p}}$.

The scalar spectral index is

$$
n_{\mathrm{S}}-\left.1 \equiv \frac{\mathrm{d} \ln A_{\mathrm{S}}^{2}}{\mathrm{~d} \ln k}\right|_{k=a H}=-6 \epsilon+2 \eta
$$

The amplitude of tensor perturbations is [16]

$$
A_{\mathrm{T}}^{2}=\left[\kappa_{4}^{2} \frac{H^{2}}{4 \pi^{2}}\right] F_{\beta}^{2}(H / \mu),
$$

where the standard expression is in square brackets, and the GB braneworld modification is defined by

$$
F_{\beta}^{-2}(x)=\sqrt{1+x^{2}}-\left(\frac{1-\beta}{1+\beta}\right) x^{2} \sinh ^{-1} \frac{1}{x} .
$$

The RS amplification factor $[6]$ is recovered when $\beta=0$. The tensor spectral index $n_{\mathrm{T}}$ is

$$
\begin{aligned}
& n_{\mathrm{T}}=\left.\frac{\mathrm{d} \ln A_{\mathrm{T}}^{2}}{\mathrm{~d} \ln k}\right|_{k=a H} \\
& =-2 \epsilon\left[1-\frac{x^{2} F_{\beta}^{2}(x)\left\{1-(1-\beta) \sqrt{1+x^{2}} \sinh ^{-1} x^{-1}\right\}}{(1+\beta) \sqrt{1+x^{2}}}\right] .
\end{aligned}
$$

The tensor-to-scalar ratio is given by

$$
R \equiv 16 \frac{A_{\mathrm{T}}^{2}}{A_{\mathrm{S}}^{2}}=-8 Q n_{\mathrm{T}}
$$

where 16

$$
\begin{aligned}
Q & =\frac{(1-\beta) \cosh \chi}{\left[1+2(1-\beta) \sinh ^{2} \frac{1}{3} \chi\right] \cosh \frac{1}{3} \chi} \\
& =\frac{1+\beta+2 \beta x^{2}}{1+\beta+\beta x^{2}}
\end{aligned}
$$

The standard form of the consistency relation, $Q=1$, $R=-8 n_{\mathrm{T}}$, holds in the absence of the Gauss-Bonnet coupling $(\beta=0)$, i.e., in both the GR and RS cases. When $\beta=0$ we have $n_{\mathrm{T}} \rightarrow-3 \epsilon$ for $x \rightarrow \infty$ (RS model) and $n_{\mathrm{T}} \rightarrow-2 \epsilon$ for $x \rightarrow 0$ (GR case). When $\beta>0$, the degeneracy factor $Q \rightarrow 2$ in the GB limit $(x \rightarrow \infty)$, thus yielding the consistency relation $R=-16 n_{\mathrm{T}}$. In this case one has $n_{\mathrm{T}} \rightarrow-\epsilon$ for $x \rightarrow \infty$. In summary:

$$
\begin{array}{lll}
n_{\mathrm{T}}=-\epsilon, \quad R=-16 n_{\mathrm{T}}=16 \epsilon & (\mathrm{GB} & \text { limit }) \\
n_{\mathrm{T}}=-3 \epsilon, \quad R=-8 n_{T}=24 \epsilon & \text { (RS limit) } \\
n_{\mathrm{T}}=-2 \epsilon, \quad R=-8 n_{T}=16 \epsilon & \text { (GR limit) }
\end{array}
$$

The behaviour of $n_{\mathrm{T}}$ is illustrated in Fig. 4

The change of the consistency relation in the GB case can lead to modification of the likelihood values of inflationary model parameters. Recently a likelihood analysis in terms of the RS inflationary parameters has been performed for the consistency relation $R=-8 n_{\mathrm{T}}$, using the latest observational data [12] (updating Ref. 11]). This likelihood analysis is the same as in GR since the consistency relation is unchanged. The same applies in generalized Einstein theories, including the 4-dimensional dilaton gravity and scalar-tensor theories 18]. The situation is different in the presence of the Gauss-Bonnet coupling.

In order to determine the difference we carried out the likelihood analysis by using a new consistency relation $R=-16 n_{\mathrm{T}}$ in the GB limit. Since the runnings of scalar and tensor perturbations are consistent with zero [3], we varied three inflationary parameters $n_{\mathrm{S}}, R$ and $A_{\mathrm{S}}^{2}$ together with four cosmological parameters by assuming a 


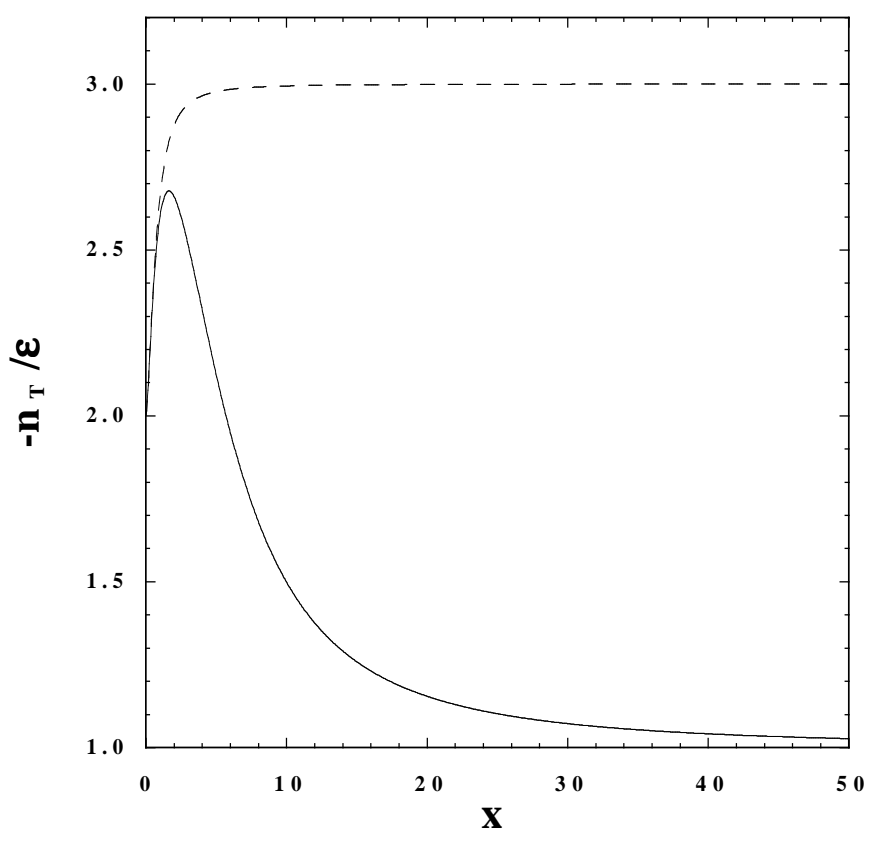

FIG. 4: The behaviour of $-n_{\mathrm{T}} / \epsilon$ as a function of the energy scale of inflation, $x=H / \mu$. The dashed curve is $\beta=0$, the solid curve is for $\beta=10^{-2}$.

flat $\Lambda$ CDM universe. We ran the Cosmological Monte Carlo (CosmoMC) code together with the CAMB program [19. In addition to the dataset from WMAP [20], the 2dF 21] and SDSS [1] galaxy redshift surveys, we implement the band powers on smaller scales from VSA 22], CBI 23] and ACBAR 24]. The 2D posterior constraints in terms of $n_{\mathrm{S}}$ and $R$ are illustrated in Fig. 5 This result is similar to those in Refs. 12, 18, which were derived using the standard consistency relation $R=-8 n_{\mathrm{T}}$. A small difference appears for the $2 \sigma$ contour bound in the region $n_{\mathrm{S}}>1$, but this is not important to constrain the inflationary models with monomial potential, Eq. (18). Thus we can safely use the contour bounds based on $R=-16 n_{\mathrm{T}}$ to constrain RS inflationary models with GB correction.

For the monomial potential, Eq. (27) gives

$$
\sigma^{(p-2) / 2 p} \beta^{(p+2) / 2 p}=\frac{2^{-1+2 / p}(2+p) \mu^{1+2 / p} \kappa_{4}^{1-2 / p} I\left(\chi_{N}\right)}{3^{(2-3 p) / 2 p} p^{2} V_{0}^{2 / p}[N(2+p)+p]} .
$$

On the other hand, applying the COBE normalization $A_{\mathrm{S}}^{2}=5 \times 10^{-9}$ in Eq. (34) yields

$$
\sigma^{1-1 / p} \beta^{2+1 / p}=\frac{3^{3-1 / p} 10^{8} \mu^{4+2 / p} \kappa_{4}^{2-2 / p}\left[\cosh \frac{2}{3} \chi_{N}-1\right]^{3}}{2^{2-2 / p} \pi^{2} p^{2} V_{0}^{2 / p}\left(\sinh \chi_{N}\right)^{2-2 / p}} .
$$

As pointed out in Ref. 15], one can determine $\sigma$ and $\beta$ for given values of $V_{0}, N, \chi_{N}$ and $p$ by using Eqs. (44)

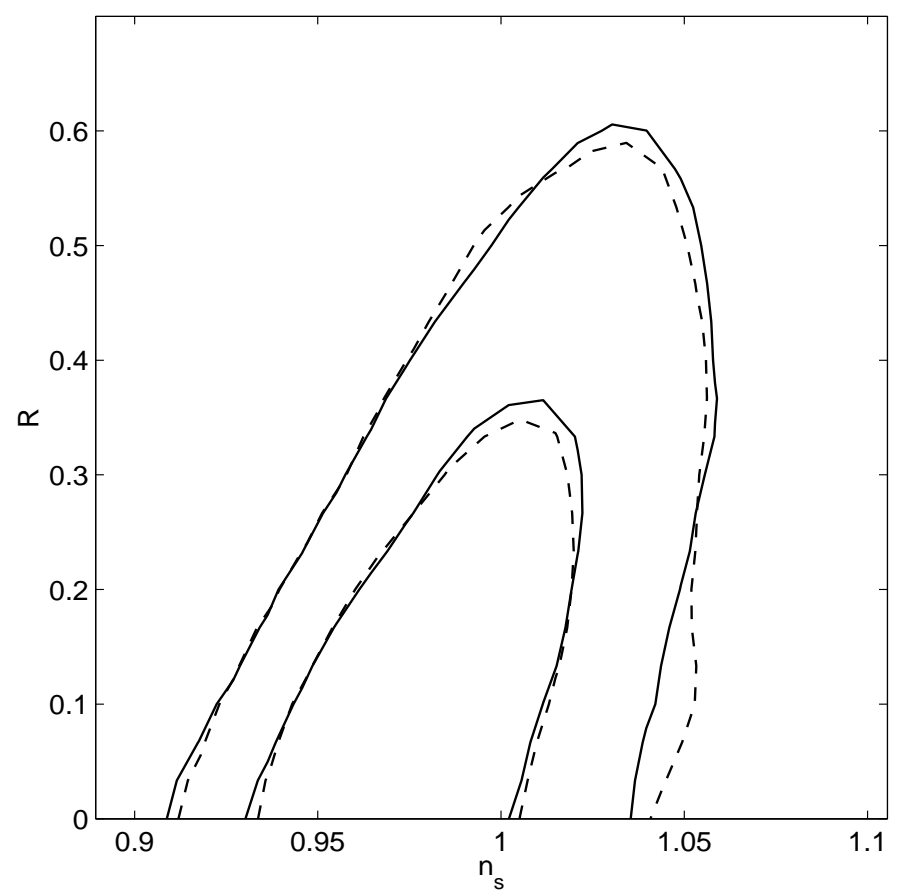

FIG. 5: 2D posterior constraints in the $n_{\mathrm{S}}-R$ plane with the $1 \sigma$ and $2 \sigma$ contour bounds. The solid curve is obtained by using the consistency relation $R=-16 n_{\mathrm{T}}$ in the GB limit, whereas the dashed curve corresponds to $R=-8 n_{\mathrm{T}}$ in the RS/GR cases.

and (45). Combining these equations, we obtain

$$
\frac{\beta^{3} \sigma}{64 \kappa_{4}^{2} \mu^{6}}=\frac{3^{3} 10^{16}}{(4 \pi)^{4}}\left[\frac{N(2+p)+p}{(2+p) I\left(\chi_{N}\right)}\right]^{2} \frac{\left[\cosh \frac{2}{3} \chi_{N}-1\right]^{6}}{\left(\sinh \chi_{N}\right)^{4-4 / p}} .
$$

This relation is illustrated in Fig. [6] We also plot the quantum gravity limit derived from Eq. (17), which may be rewritten as

$$
48 \sinh ^{6} \chi<(8 \pi)^{8}\left(\frac{\beta^{3} \sigma}{64 \kappa_{4}^{2} \mu^{6}}\right),
$$

where we have used Eqs. (21), (6) and (7). We find the upper limits $\chi_{N}<11.65$ for $p=2$, $\chi_{N}<11.22$ for $p=4$ and $\chi_{N}<10.31$ for $p \rightarrow \infty$. This information is important to place limits on the values of $n_{\mathrm{S}}$ and $R$.

By Eqs. (28), (29), (35), (38), (39) and (40) one can evaluate the spectral index $n_{\mathrm{S}}$ and the ratio $R$ as a function of $\chi_{N}$ for given values of $N$ and $p$. In Fig. 7 we plot $n_{\mathrm{S}}$ for $N=60$. When $p=2$ and $p=4$ the spectral index has a maximum at $\chi_{N} \sim 4$. This is associated with the fact that the slow-roll parameter $\epsilon$ has a minimum in the intermediate region (see Fig. 2). Although $\eta$ is an increasing function of $\chi_{N}$, the effect of $\epsilon$ is more important in $n_{\mathrm{S}}$. The spectral index $n_{\mathrm{S}}$ decreases for larger $\chi_{N}$ in the GB region because of the increase of $\epsilon$. In the case of the exponential potential $(p \rightarrow \infty), n_{\mathrm{S}}$ is a monotonically increasing function. It was shown in Ref. [15] that one gets an exactly scale-invariant spectrum, $n_{\mathrm{S}}=1$, for 


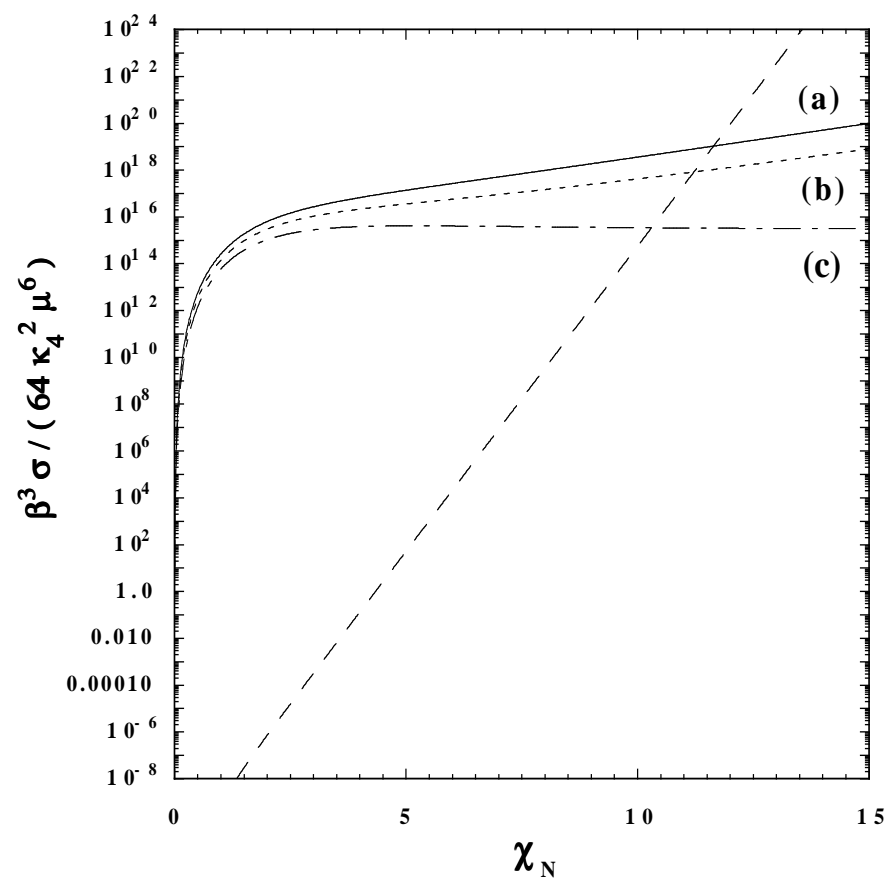

FIG. 6: The dependence of the quantity $\beta^{3} \sigma / 64 \kappa_{4}^{2} \mu^{6}$ on $\chi_{N}$ for (a) $p=2$, (b) $p=4$, (c) $p \rightarrow \infty$. We also show the quantum gravity limit curve, Eq. (47). The allowed region is above and to the left of this curve.

the background evolution characterized by $H^{2} \propto \rho^{2 / 3}$. Therefore the spectral index approaches $n_{\mathrm{S}}=1$ in the GB limit $\left(\sinh \chi_{N} \gg 1\right)$.

Figure 8 shows the behaviour of the tensor to scalar ratio $R$, determined by Eqs. (38) and (40). We find that the exact results for $\chi_{N} \gtrsim 4$ are well approximated by the relation $R=16 \epsilon$ which holds in the GB limit. The difference appears in the RS regime, where the consistency relation is $R=24 \epsilon$. The ratio $R$ has a minimum around $\chi_{N} \sim 2$, depending on the value of $p$. This is again linked to the fact that the slow-roll parameter $\epsilon$ has a minimum in this intermediate region. Beyond this region, $R$ is an increasing function of $\chi_{N}$ (limited by the quantum gravity constraint). When $p \rightarrow \infty$ the value of $R$ becomes too large for the observational contour bounds, as we see later.

The values of $n_{\mathrm{S}}$ and $R$ in the RS limit are 12

$$
n_{\mathrm{S}}-1=-\frac{2(2 p+1)}{N(p+2)}, \quad R=\frac{24 p}{N(p+2)} .
$$

These agree with our results shown in Figs. 17 and 8 in the RS regime. The perturbations in the RS case tend to be further from the point $\left(n_{\mathrm{S}}=1, R=0\right)$ relative to the standard GR case for small exponents $p$ [11]. This is mainly due to the fact that $\epsilon$ is larger in the RS case. A similar feature arises in the intermediate regime between RS and GB. Since the background equation changes from $H^{2} \propto \rho^{2}$ (RS) to $H^{2} \propto \rho^{2 / 3}(\mathrm{~GB})$ with the growth of $\chi_{N}$, there is an intermediate region in which the background

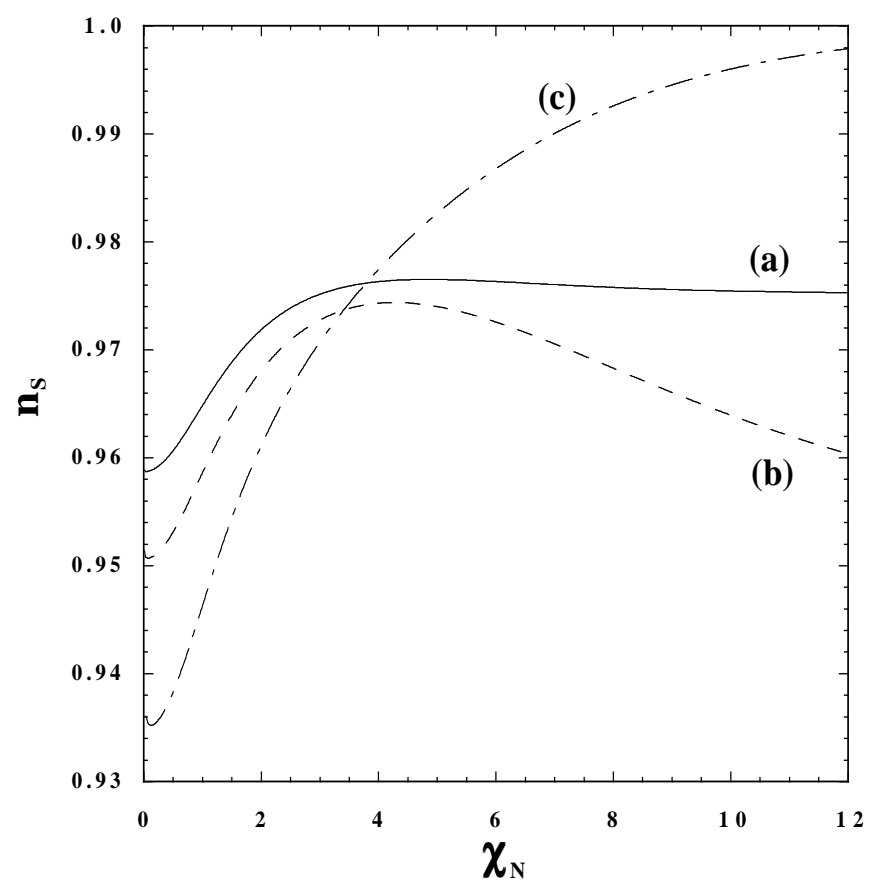

FIG. 7: The spectral index $n_{\mathrm{S}}$ as a function of $\chi_{N}$ when $N=60$, for (a) $p=2$, (b) $p=4$, (c) $p \rightarrow \infty$.

is characterized by $H^{2} \propto \rho$. This leads to the minimum of $R$ seen in Fig. 8

\section{OBSERVATIONAL CONSTRAINTS}

By plotting the theoretical values of $n_{\mathrm{S}}$ and $R$ in the $2 \mathrm{D}$ posterior observational contour bounds of Fig. [5] we can constrain the GB inflationary models with monomial potential. The parametric theoretical curves $\left(n_{\mathrm{S}}\left(\chi_{N}\right), R\left(\chi_{N}\right)\right)$ are plotted for values of $\chi_{N}$ up to the quantum gravity limit $\chi_{N} \approx(11.65,11.22,10.31)$, for $p=(2,4, \infty)$ respectively.

\section{- $V \propto \phi^{2}$}

In the RS case, the quadratic potential $(p=2)$ is inside the $2 \sigma$ contour for $N=50$, and marginally on the $1 \sigma$ contour bound for $N=60$, as seen from the left edge point of the curve (a) in Figs. 9 and 10, This RS inflationary model is thus observationally allowed for $N \gtrsim 50$ [1, 12]. As seen in Fig. $7 n_{\mathrm{S}}$ for $\beta>0$ gets closer to scale-invariance than the RS limiting value. In addition, Fig. 8 indicates that $R$ is smaller than in the RS case. Therefore the theoretical points tend to move deeper inside the observationally allowed region, as shown in Figs. 9 and 10. The RS limiting case is marginally allowed for $N=50$, but the GB coupling moves the points inside the $1 \sigma$ contour bound. Thus the quadratic potential is observationally more favoured when the GB term is present. 


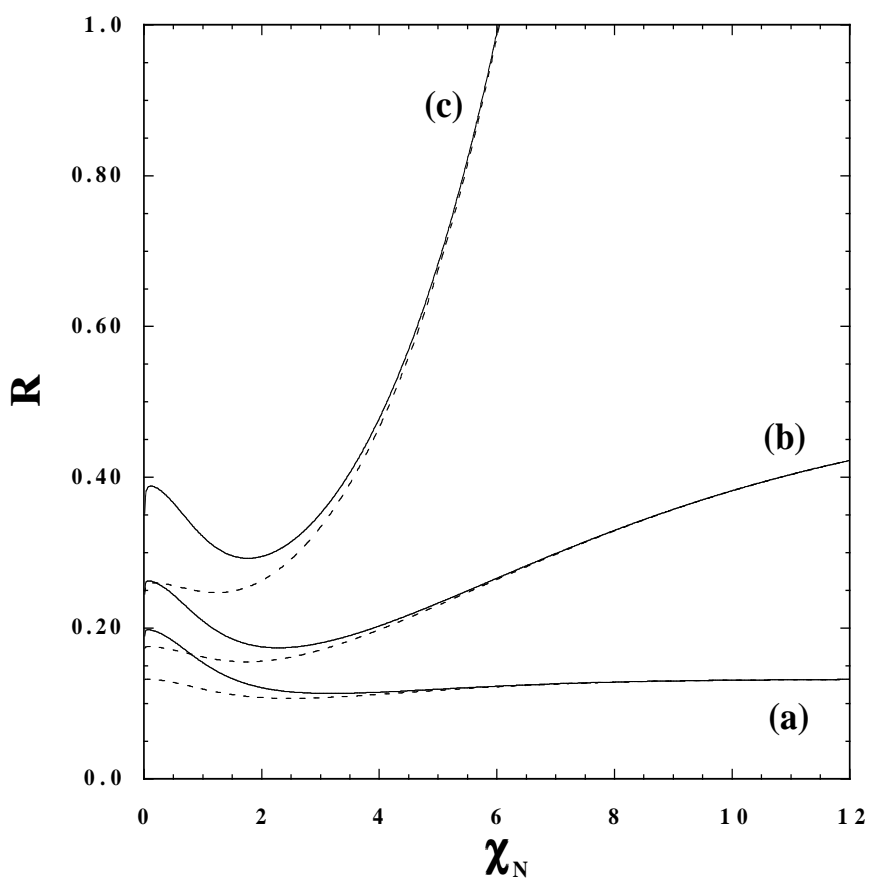

FIG. 8: The tensor to scalar ratio $R$ as a function of $\chi_{N}$ when $N=60$, for (a) $p=2$, (b) $p=4$, (c) $p \rightarrow \infty$ (solid curves). The dotted curves are obtained from $R=16 \epsilon$, which only holds in the GB limit.

\section{- $V \propto \phi^{4}$}

The quartic potential ( $p=4)$ is under strong observational pressure in both RS [1, 12] and GR 2, 3] cases. In fact in the RS case it is outside the $2 \sigma$ contour bound for $N<60$, as seen in the left edge point of the curve (b) in Fig. 9] The GB term leads to an increase of $n_{\mathrm{S}}$ and decrease of $R$ in the intermediate regime between RS and GB. As in the case $p=2$, this property improves the observational compatibility of the GB brane-world. However in the extreme GB limit for the quartic potential, the opposite happens, and the theoretical points move back into the RS region.

In summary, the GB term rescues the quartic potential as an observationally allowed model, for inflationary energy scales in the intermediate regime.

\section{- $V=V_{0} \exp \left(\xi \kappa_{4} \phi\right)$}

Here $\xi$ is a dimensionless constant. The discussion in Secs. II and III can be applied by taking the limit $p \rightarrow$ $\infty$ and making the replacement $p^{2} V_{0}^{2 / p} \mapsto \xi^{2} \kappa_{4}^{2}$. In the RS case the tensor to scalar ratio is too large to be compatible with observations [11], as seen in left edge point of curve (c) in Figs. 9] and [10] We require $N \gtrsim 90$ in order for the RS point to be inside the $2 \sigma$ bound, but this is beyond cosmologically relevant scales.

The GB term works in a similar way to the previous cases. There is a minimum value of $R$ in the intermediate

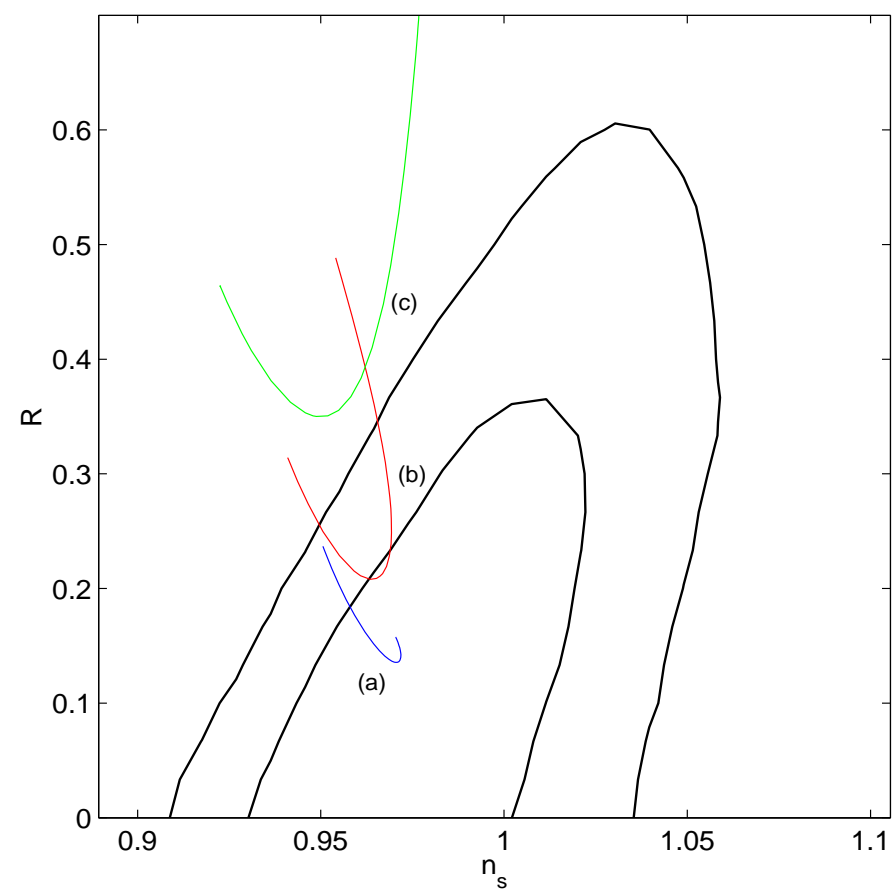

FIG. 9: Theoretical prediction of Gauss-Bonnet monomial inflation models for $N=50$, together with the $1 \sigma$ and $2 \sigma$ observational contour bounds. The left edge point of the theoretical curves is the RS limit, $\chi_{N} \ll 1$, and $\chi_{N}$ increases along the curves up to the quantum gravity limit. The curves are shown for (a) $p=2$, (b) $p=4$, (c) $p \rightarrow \infty$.

regime between $\mathrm{RS}$ and GB. For $N=50$ this effect is not sufficient: as seen in Fig. 9] the theoretical curve is closer but still outside the $2 \sigma$ contour bound. However some of the theoretical points move inside the $2 \sigma$ bound for $N \gtrsim 55$, as shown in Fig. [10] Then steep inflation is not necessarily ruled out, provided that $\chi_{N}$ corresponds to an intermediate energy scale, between RS and GB. Note that $R$ rapidly grows with $\chi_{N}$ in the GB regime. Figure 8] shows that for $N=60, R=1.00$ at $\chi_{N} \approx 6$. Steep inflation in the GB regime is disfavoured due to the large tensor to scalar ratio, in spite of the fact that the spectral index $n_{\mathrm{S}}$ approaches 1 .

\section{CONCLUSIONS}

In this paper we have studied the observational constraints on Gauss-Bonnet brane-world inflation, using the latest observational datasets. The presence of the GB term as a correction to the RS model modifies the standard consistency relation, $R=-8 n_{\mathrm{T}}$. We carried out a likelihood analysis in terms of inflationary and cosmological parameters by using the GB consistency relation, $R=-16 n_{\mathrm{T}}$, which holds in the high-energy GB limit. The likelihood result is almost identical to the one in the standard case, which means that this can be safely used even in an intermediate regime between GB and RS. 


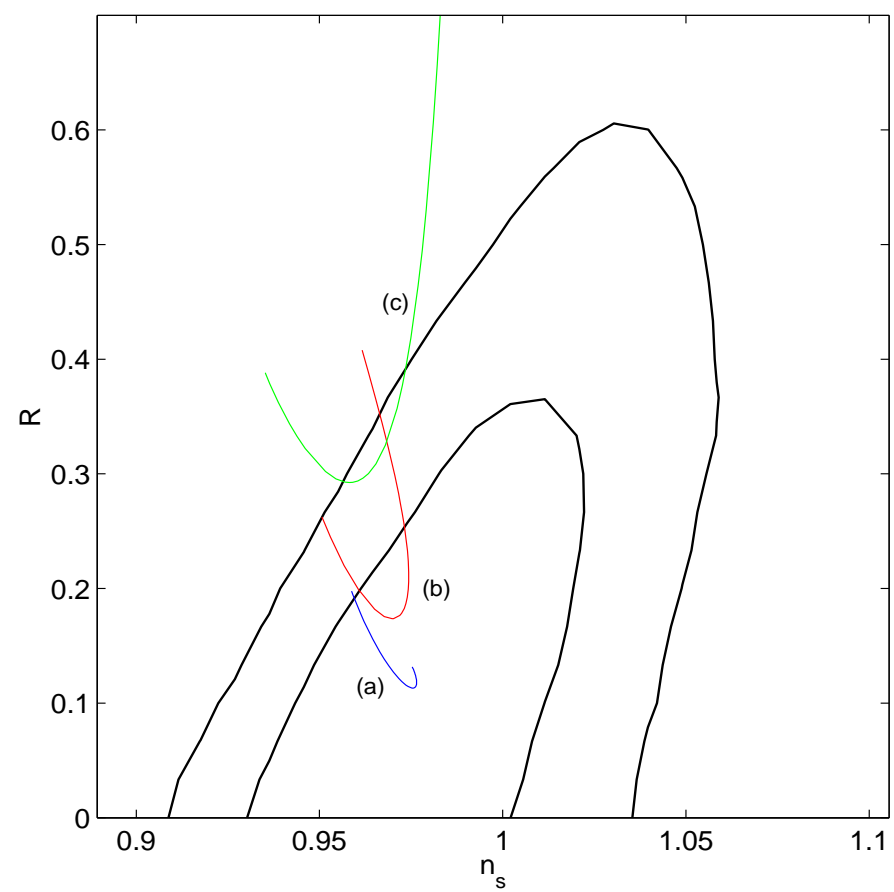

FIG. 10: As in Fig. 9] with $N=60$.

We have considered monomial potentials, Eq. [18), including the exponential potential as a limiting case. The slow-roll parameters $\epsilon$ and $\eta$ are evaluated as functions of the dimensionless energy scale $\chi_{N}$ and the e-folds $N$ [see Eqs. (28) and (29)]. An important feature we found is that $\epsilon$ has a minimum in the intermediate region between the GB and RS limits, which particularly affects the ratio $R$ of tensor to scalar perturbations. We evaluated $R$ and the spectral index $n_{\mathrm{S}}$ of scalar perturbations as functions of $\chi_{N}$. Then we plotted these theoretical values together with the $2 \mathrm{D}$ observational constraints in the $n_{\mathrm{S}}-R$ plane. As seen in Fig. $8, R$ has a minimum for intermediate energies, irrespective of the values of $p$. In addition $n_{\mathrm{S}}$ is a growing function for $\chi_{N} \lesssim 2-3$. Therefore in this region we obtain smaller $R$ and larger $n_{\mathrm{S}}$ than in the RS case. This feature of GB brane-world inflation improves the observational compatibility of the 3 potentials considered, relative to the RS case.

For the quadratic potential $(p=2)$, we found that the theoretical points move inside the $1 \sigma$ contour bound for $N=50$, while the RS point is marginally inside of the $2 \sigma$ bound (see Fig. 9). This means that the quadratic potential is favoured observationally even for rather low e-folds in the presence of the GB term.

It is known that the quartic potential $(p=4)$ is under strong observational pressure both in the RS and GR cases, but this situation is improved by a GB correction term. Figure 9 shows that there exists a region of parameter space which is inside the $2 \sigma$ bound even for $N=50$.

The exponential potential $(p \rightarrow \infty)$ is ruled out in the RS case. However the GB term allows smaller values of $R$, thereby giving rise to some regions which are inside the $2 \sigma$ bound for $N \gtrsim 55$ (see Fig. 10). This is an intriguing possibility to save the brane-world steep inflation models (e.g., Ref. [25]). It may also be of interest to extend our analysis to the case in which the system is described by the Dirac-Born-Infeld-type action on the brane, since steep potentials of the type $V(\phi) \propto \exp \left(m^{2} \phi^{2}\right)$ appear in such a theory [26].

Acknowledgments: We are grateful to Sam Leach for providing the latest SDSS code and Antony Lewis and David Parkinson for technical help in numerics. The work of RM is supported by PPARC.
[1] D. N. Spergel et al., Astrophys. J. Suppl. 148, 175 (2003) arXiv:astro-ph/0302209; M. Tegmark et al. [SDSS Collaboration], Phys. Rev. D69, 103501 (2004) arXiv:astro-ph/0310723.

[2] H. V. Peiris et al., Astrophys. J. Suppl. 148, 213 (2003); V. Barger, H. S. Lee, and D. Marfatia, Phys. Lett. B565, 33 (2003) arXiv:hep-ph/0302150; W. H. Kinney, E. W. Kolb, A. Melchiorri, and A. Riotto, arXiv:hep-ph/0305130

[3] S. M. Leach and A. R. Liddle, Phys. Rev. D68, 123508 (2003) arXiv:astro-ph/0306305.

[4] P. Brax and C. van de Bruck, Class. Quantum Grav. 20, R201 (2003) arXiv:hep-th/0303095; R. Maartens, Liv. Rev. Rel., to appear (2004) arXiv:gr-qc/0312059; P. Brax, C. van de Bruck, and A-C. Davis, arXiv:hep-th/0404011

[5] R. Maartens, D. Wands, B. A. Bassett and I. Heard, Phys. Rev. D62, 041301 (2000) arXiv:hep-ph/9912464.

[6] D. Langlois, R. Maartens, and D. Wands, Phys. Lett.
B489, 259 (2000) arXiv:hep-th/0006007.

[7] G. Huey and J. E. Lidsey, Phys. Lett. B514, 217 (2001) arXiv:astro-ph/0104006; D. Seery and A. Taylor, arXiv:astro-ph/0309512

[8] G. Calcagni, JCAP 0311, $009 \quad$ (2003) arXiv:hep-ph/0310304; ibid., arXiv:hep-ph/0312246 E. Ramirez and A. R. Liddle, Phys. Rev. D 69, 083522 (2004) arXiv:astro-ph/0309608.

[9] A. R. Liddle and A. N. Taylor, Phys. Rev. D65, 041301 (2002) arXiv:astro-ph/0109412.

[10] K. Koyama, Phys. Rev. Lett. 91, 221301 (2003) arXiv:astro-ph/0303108; C. S. Rhodes, C. van de Bruck, Ph. Brax, A. C. Davis, Phys. Rev. D68, 083511 (2003) arXiv:astro-ph/0306343.

[11] A. R. Liddle and A. J. Smith, Phys. Rev. D68, 061301 (2003) arXiv:astro-ph/0307017.

[12] S. Tsujikawa and A. R. Liddle, JCAP 03, 001 (2004) arXiv:astro-ph/0312162.

[13] C. Charmousis and J. F. Dufaux, Class. Quant. Grav. 19, 
4671 (2002) arXiv:hep-th/0202107; S. C. Davis, Phys. Rev. D67, 024030 (2003) arXiv:hep-th/0208205; P. Binetruy, C. Charmousis, S. C. Davis and J. F. Dufaux, Phys. Lett. B544, 183 (2002) arXiv:hep-th/0206089.

[14] K. Maeda and T. Torii, Phys. Rev. D69, 024002 (2004) arXiv:hep-th/0309152.

[15] J. E. Lidsey and N. J. Nunes, Phys. Rev. D67, 103510 (2003) arXiv:astro-ph/0303168

[16] J. F. Dufaux, J. E. Lidsey, R. Maartens and M. Sami, arXiv:hep-th/0404161

[17] E. J. Copeland, A. R. Liddle, and J. E. Lidsey, Phys. Rev. D64, 023509 (2001) arXiv:astro-ph/0006421.

[18] S. Tsujikawa and B. Gumjudpai, Phys. Rev. D 69, 123523 (2004) arXiv:astro-ph/0402185.

[19] A. Lewis, A. Challinor, and A. Lasenby, Astrophys. J. 538, 473 (2000) arXiv:astro-ph/9911177; A. Lewis and S. Bridle, Phys. Rev. D66, 103511 (2002) arXiv:astro-ph/0205436; see also http://camb.info/
[20] http://lambda.gsfc.nasa.gov/

[21] W. J. Percival et al., Mon. Not. Roy. Astron. Soc. 327, 1297 (2001) arXiv:astro-ph/0105252.

[22] K. Grainge et al., Mon. Not. Roy. Astron. Soc. 341, L23 (2003) arXiv:astro-ph/0212495.

[23] T. Pearson et al., Astrophys. J. 591, 556 (2003) arXiv:astro-ph/0205388.

[24] C. L. Kuo et al., Astrophys. J. 600, 32 (2004) arXiv:astro-ph/0212289.

[25] V. Sahni, M. Sami and T. Souradeep, Phys. Rev. D 65, 023518 (2002) arXiv:gr-qc/0105121; M. Sami and V. Sahni, arXiv:hep-th/0402086

[26] M. R. Garousi, JHEP 0312, $036 \quad$ (2003) arXiv:hep-th/0307197; M. R. Garousi, M. Sami and S. Tsujikawa, arXiv:hep-th/0402075 ibid., arXiv:hep-th/0405012 